\title{
Array Sensing Using Electromagnetic Method for Detection of Smelting in Submerged Arc Furnaces
}

\author{
WeiLing Liu ${ }^{1}$, XiaoHong Han $^{2}$, LingZhen Yang ${ }^{3}$, and XiaoMing Chang ${ }^{1 *}$ \\ ${ }^{1}$ College of Computer Science and Technology, Taiyuan University of Technology, No. 79 Yingze West Street, \\ Taiyuan 030024, Shanxi, PR China \\ ${ }^{2}$ College of Mechanical Engineering, Taiyuan University of Technology, No. 79 Yingze West Street, Taiyuan 030024, Shanxi, PR China \\ ${ }^{3}$ College of Physics and optoelectronics, Taiyuan University of Technology, No. 79 Yingze West Street, Taiyuan 030024,
}

Shanxi, PR China

(Received 30 May 2016, Received in final form 13 July 2016, Accepted 22 July 2016)

\begin{abstract}
In this paper, we propose an array sensing detection method for smelting of submerged arc furnaces (SAF) based on electromagnetic radiation. AC magnetic field generated by electrode currents and molten currents in the furnace is reflected outside of the furnace. According to the spatial distribution of electromagnetic field a radiation model of SAF is built. We design a 3D magnetic field sensing array system in order to collect the magnetic field information. Through the collected information, the current distribution characteristics of SAF are described and the key parameters of smelting are obtained. Theoretical simulation and field test show that the curves acquired by the sensing array can accurately reflect the information of the relative displacement when the relative displacement between the array and electrode is $10 \mathrm{~cm}$. Compared with the detection method of 3D single point, the proposed array sensing method of magnetic field obtains better results in terms of realtime and accuracy, and has good practical value for industrial measurement.
\end{abstract}

Keywords : submerged arc furnace, key parameters of smelting, magnetic field, array sensing

\section{Introduction}

Submerged arc furnace (SAF) is an industrial electric furnace which gets power through graphite electrodes. The electric arcs emerge between the furnace burden and the electrode tip, which makes the electrical energy change into heat energy. The obtained heat energy can be used to melt ores. By smelting the ores many types of ferroalloys can be obtained such as ferrosilicon, ferromanganese, ferrochrome, ferrotungsten, and silicon-manganese alloys, and so on [1-3]. However, a typical SAF consumes a great deal of electrical energy (approximately $450 \mathrm{kWh} /$ ton) in the process of smelting. Therefore, it is necessary to study the SAF smelting processes from the economic and environmental points of view [4-6].

Smelting in SAF is an extremely complicated process involving physical-chemical reactions and heat exchanges [7-9]. The fluctuation of the furnace condition occurs

(C)The Korean Magnetics Society. All rights reserved.

*Corresponding author: Tel: 132-3369-7523

Fax: 0351-6014033, e-mail: 644855751@qq.com frequently under the influence of many factors. Thus measurement technique and detection system for the furnace are necessary for operators to analyze the furnace condition accurately and take measures opportunely to guarantee the stable running of the furnace $[10,11]$. In practice, the furnace condition is observed through simple instruments, personal experiences or naked eyes, which are difficult to get the optimal smelting parameters and thus lower production efficiency [12]. To overcome the drawbacks, lots of methods have been put forward to measure these key parameters. However some of these methods get the measurement through indirect calculation and speculation [13-16]. Other methods only get the position of electrode tip roughly $[17,18]$.

We propose a non-contact array sensing detection method of magnetic field. Based on the inner structure of SAF and electromagnetism, a magnetic field radiation model of SAF is established. We analyze the problems existing in the 3D single point detection method of magnetic field and then propose a model of array detection. The spatial distance of the detection points is calculated and determined through probing the distributing rule of 
magnetic field in SAF. The theoretical analysis and preliminary experiments show that the proposed method gets better results in real-time and precision compared with the single point 3D detection method. Using our proposed method, the maintenance of the equipment is more convenient in later periods than the single point $3 \mathrm{D}$ detection method.

The rest of paper is organized as follows. Section 2 describes the measuring principle and system structure. In Section 3, determination of the spatial distance of detection points is introduced. Thereafter experimental results are presented in Section 4, and conclusions are given in Section 5 .

\section{Measuring Principle and System Structure}

\subsection{Magnetic field radiation model}

The hearth structure of SAF is shown in Fig. 1 [19-21].

The electric arc is generated between electrode tip and molten bath shown in Fig. 1. The molten alloys gather in molten bath, and the top of molten bath surface, is the position of liquid level.

Through the electrodes, large currents flow into the furnace, in which the circuit can be regarded as two types of loop: star circuit loop and triangle circuit loop [22, 23], and the two types of loop can be equivalently transformed. In this paper we take the triangle loop as an example to analyze the circuit model. Based on electromagnetic

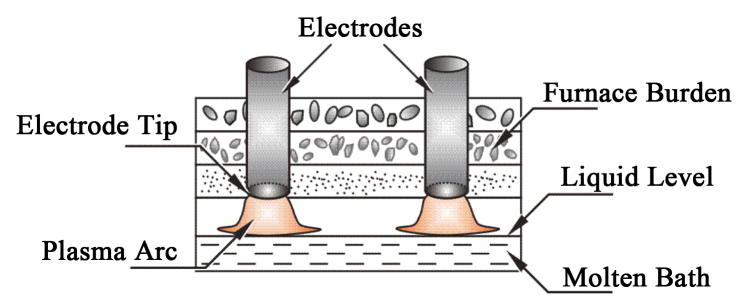

Fig. 1. (Color online) The hearth structure of SAF.

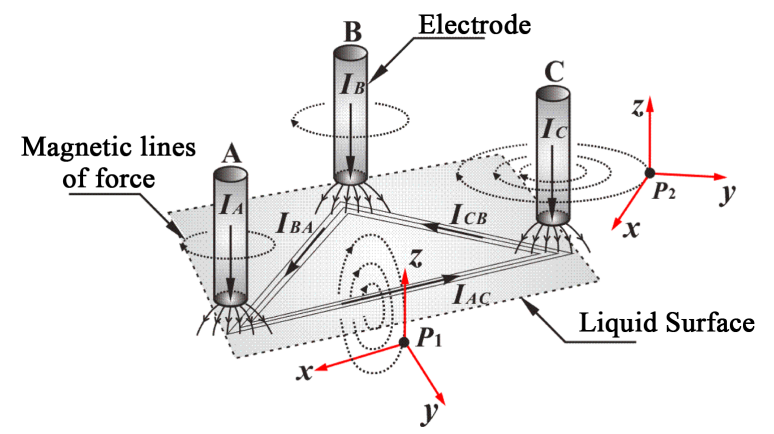

Fig. 2. (Color online) The magnetic field radiation model of SAF.

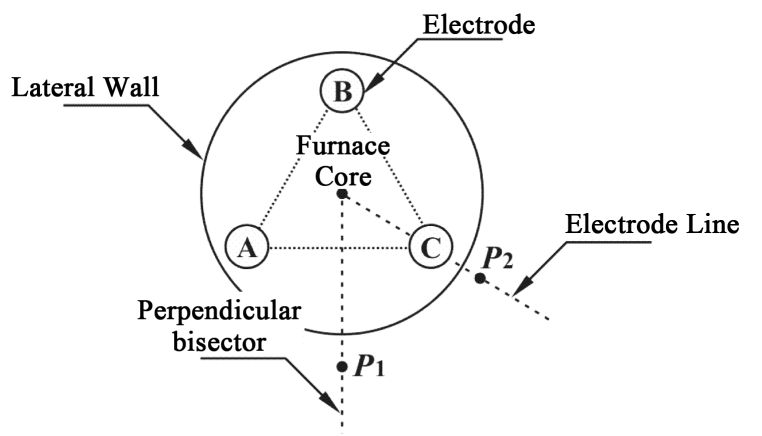

Fig. 3. The plan view of the SAF.

principles, magnetic field radiation model of SAF can be built, as shown in Fig. 2.

In Fig. 2, the electrode currents $I_{A}, I_{B}, I_{C}$ flow into the furnace along each electrode and near the liquid surface they form a triangle circuit loop composed of the currents $I_{A C}, I_{C B}, I_{B A}$. The two points $P_{1}$ and $P_{2}$ are the measured origins, respectively. $I_{A C}$ is the molten bath current parallel to the $x$-axis component $B_{x}$ at point $P_{1} . I_{C}$ is the electrode current parallel to the $z$-axis component $B_{z}$ at point $P_{2}$. In order to describe conveniently, the concepts of electrode line and perpendicular bisector are defined as shown in Fig. 3.

In Fig. 3 the plan view of SAF is given. On the plane parallel to the molten bath surface, a regular triangle is generated through connecting three vertices of electrodes, and the core of SAF is the center of the triangle. The line connecting the electrode and the furnace core is called electrode line. The vertical of each edge of the regular triangle is termed as perpendicular bisector. It can be seen that the perpendicular bisector is parallel to $y$-axis component $B_{y}$ of point $P_{1}$, and the electrode line is parallel to $y$-axis component $B_{y}$ of point $P_{2}$.

\subsection{D single point detection model}

The 3D single point detection system of magnetic field shown in Fig. 4 is composed of 3D probe, main control unit (MCU), and PC.

In Fig. 4, the upper PC gives the control commands, and MCU collects and processes the 3D probe magnetic

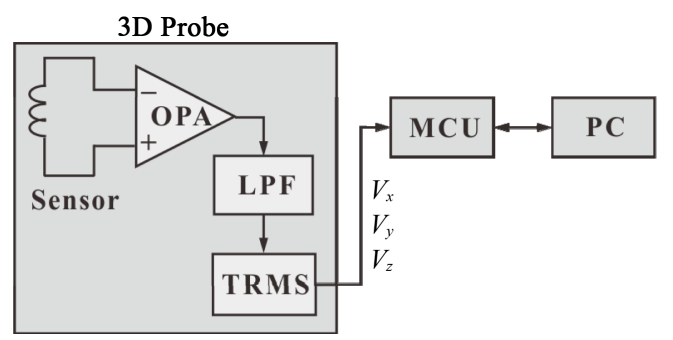

Fig. 4. The architecture of $3 \mathrm{D}$ single point detection system. 
field information and sends the information back to the PC. 3D probe is composed of the magnetic sensor, operational amplifier (OPA), low-pass filter (LPF), and true RMS conversion circuit (TRMS).

For any system in which high real-time is demanded, the delay time caused by information transmission is unavoidable and cannot be ignored [24]. For the 3D single point detection system, sampling and processing time $T_{S P}$ is analyzed and expressed as Eq. (1).

$$
T_{S P}=\sum_{i=1}^{5} T_{S i}
$$

In Eq. (1), $T_{S 1}, T_{S 2}, T_{S 3}, T_{S 4}, T_{S 5}$ are the time of sensor collection, signal amplification, low-pass filtering, TRMS conversion and MCU processing, respectively. In the practical engineering problems, $T_{S 1}, T_{S 2}, T_{S 3}$ and $T_{S 5}$ are all in milliseconds level. However, TRMS conversion circuit adopts the principle of integral, and the higher the output accuracy is required, the longer the processing time that TRMS conversion takes will be. Generally, the time, which it takes for the TRMS conversion circuit to convert once until to stable states, $T_{S 4}$, is approximately equal to $T_{S 4} \approx 3 \sim 5 \mathrm{~s}$. If only taking the inflence of the TRMS conversion time $T_{S 4}$ into account, the single sampling and processing time $T_{S P}$ is approximately equal to $T_{S P} \approx T_{S 4} \approx 3 \sim 5 \mathrm{~s}$.

In the 3D single point detection way of magnetic field, to attain the key parameters in the furnace, the data of several detection points needs to be acquired. Namely when the sampling work of every point is finished, the $3 \mathrm{D}$ probe needs to be moved to next detection point. To sample $N$ points using the 3D single point detection system, the time $T_{N S}$ is presented as Eq. (2).

$$
T_{N S}=N \times T_{S P}+(N-1) \times T_{M O V}
$$

where $N$ is the number of sampling points. $T_{M O V}$ is the moving time between adjoining sampling points of the 3D single point detection system. For the practical engineering problems, $T_{M O V} \geq 3 \mathrm{~s}$.

In conclusion, the minimum values of the sampling and processing time $T_{S P}$ and the moving time $T_{M O V}$ are approximately equal to $T_{S P(\min )} \approx 3 \mathrm{~s}$ and $T_{M O V(\min )} \approx 3 \mathrm{~s}$, respectively. We apply the parameters mentioned above to Eq. (2) to get the minimum value of the time $T_{N S}$ which is given as Eq. (3).

$$
T_{N S(\min )} \approx 6 N-3
$$

From Eq. (3), the collecting time $T_{N S}$ increases with the number of sampling points. When the total measurement length is $150 \mathrm{~cm}$ and sampling spatial distance is $10 \mathrm{~cm}$, the number of sampling points is 15 . According to Eq.
(3), the collecting time $T_{N S}$ is at least $87 \mathrm{~s}$. However, during the period of $87 \mathrm{~s}$, the furnace condition may have changed greatly, so this detection way can't truly reflect the furnace condition. Moreover, there still exist many kinds of errors in the 3D single point detection method, and the factors which produce errors mainly include the following aspects:

(1) Due to the trembling of the mechanical equipments, it is difficult to keep measuring baseline and datum plane united in the process of moving.

(2) For moving mechanical equipments, it is difficult to keep uniformity of detection points for a long time.

(3) Moving mechanically will increase the failure rate of testing parts of the system.

(4) Considering the measuring accuracy and the maintenance of equipment in later periods, more complexity in mechanical construction design is required.

Due to the above limitations, the 3D single point detection system of magnetic field is difficult to satisfy the measurement demand in the real industrial field.

\subsection{Modelling the array sensing detection}

To improve the performance in terms of real-time and precision, combining with the principle of datum plane unity in the theory of instrument precision [25], we propose an array sensing detection method of magnetic field, and the system constitution is shown in Fig. 5.

In Fig. 5, 3D magnetic array is composed of $N$ 3D probes arranged in a linear and uniform way and every probe is equipped with a sub-microcontroller (S_MCU). When main-microcontroller (M_MCU) receives the commands from PC and triggers $N$ S_MCUs simultaneously, the $N$ S_MCUs will collect the three-dimensional magnetic field data using the $3 \mathrm{D}$ probes and send the information back to PC.

From Fig. 5, there is no moving time in the array sensing detection way. When $N$ S_MCUs gather the data simultaneously, the delay time $T_{N A}^{-}$is equal to the sampling and processing time of $3 \mathrm{D}$ single point detection system $T_{S P}$, as shown in Eq. (4).

$$
T_{N A} \approx T_{S P}
$$

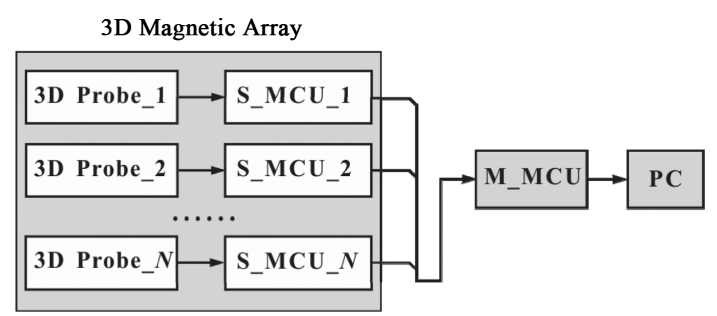

Fig. 5. The constitution of the array sensing detection system of magnetic field. 
From Eq. (4), the acquisition time of array sensing detection system is equal to $T_{N A} \approx 3 \sim 5 \mathrm{~s}$, and is independent of the number of sampling points. Therefore, the real-time performance of array sensing detection is far better than single point detection. Moreover, there does not exist the moving problem of mechanical equipment in the array detection way, which guarantees the homogeneity of the sampling interval and the unity of measuring baseline and datum plane. Our proposed method simplifies the design of mechanical connection structure and makes the maintenance of the equipment easy in later periods. So the testing precision and collection efficiency is greatly improved.

\section{Design of Array Sensor Structure}

\subsection{Modelling the magnetic field distribution}

The detected point $P_{1}$ in Fig. 2 is assumed to be the origin $o$, and several detection points $\left(S_{1}, S_{2}, \ldots, S_{\mathrm{m}}, \ldots\right.$, $\left.S_{\mathrm{i}}\right)$ are chosen from the $z$-axis. In these detection points, the point $S_{\mathrm{m}}$, which overlaps with the point $P_{1}$, locates on the geometric plane generated by current $I_{A C}, I_{C B}$, and $I_{B A}$. The magnetic induction intensity distribution at point $P_{1}$ in perpendicular bisector is shown in Fig. 6 under the influence of the magnetic field produced by current $I_{A C}$.

From Fig. 6 the relation between the distance $r$ from the detection point $S_{\mathrm{i}}$ to the current $I_{A C}$ and the height of point $S_{\mathrm{i}}$ is presented in Eq. (5):

$$
r^{2}=h^{2}+a^{2}
$$

Where $h_{i}$ is the height of detection point $S_{\mathrm{i}} ; a$ is the distance from the origin $o$ to the pathway of current $I_{A C}$. Because the detection point $S_{\mathrm{i}}$ is at the perpendicular bisector, the distance from point $S_{\mathrm{i}}$ to the starting point of current $I_{A C}$ equals the distance from point $S_{\mathrm{i}}$ to the end point which is given by Eq. (6).

$$
R=\sqrt{\left(\frac{L_{A C}}{2}\right)^{2}+r^{2}}
$$

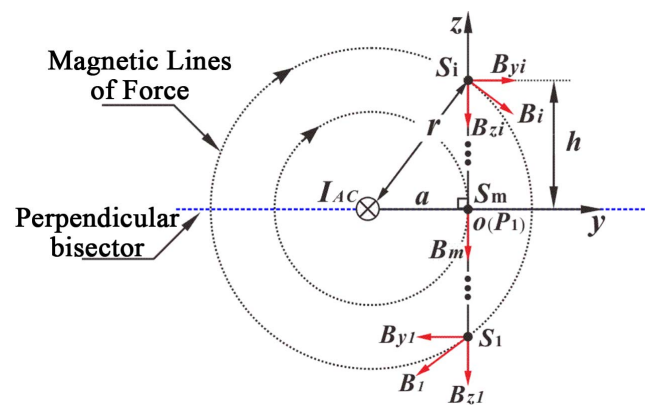

Fig. 6. (Color online) The diagram of magnetic induction intensity distribution of the current $I_{A C}$.

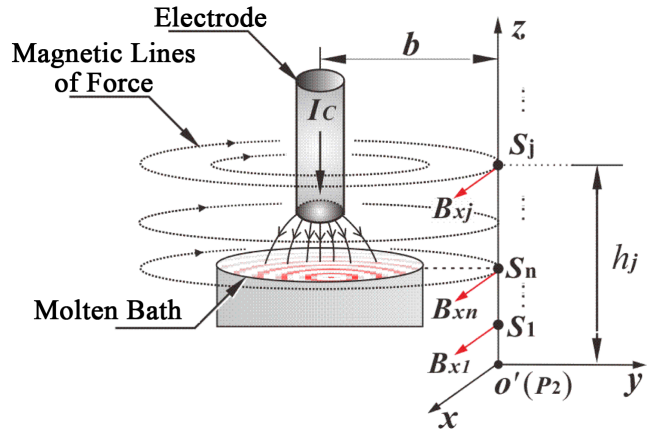

Fig. 7. (Color online) The diagram of magnetic induction intensity distribution at detected point $P_{2}$.

Where $L_{A C}$ is the length of the straight wire carrying current $I_{A C}$.

From Fig. 6, it can be seen that the magnetic flux density components of the detection point $S_{\mathrm{i}}$ along the $x, y$ and $z$-axis are $B_{x j}, B_{y i}$, and $B_{z i}$ which are given as Eq. (7).

$$
\left\{\begin{array}{l}
B_{y}=\frac{\mu_{0} I_{A C} L_{A C} h}{4 \pi R r^{2}} \\
B_{x}=0 \\
B_{z}=\frac{\mu_{0} I_{A C} L_{A C} a}{4 \pi R r^{2}}
\end{array}\right.
$$

The detected point $P_{2}$ in Fig. 2 is assumed to be the origin $o^{\prime}$, and several detection points $\left(S_{1}, \ldots, S_{\mathrm{n}}, \ldots, S_{\mathrm{j}} \ldots\right)$ are chosen on the $z$-axis. $h_{j}$ is the height of the point $S_{\mathrm{j}}$, and $h_{n}$ is the height of the molten bath surface. The magnetic induction intensity distribution at point $P_{2}$ on electrode line is shown in Fig. 7 under the influence of the magnetic field produced by the current $I_{C}$.

From Fig. 7, the magnetic flux density components of the detection point $S_{\mathrm{j}}$ along the $x, y$ and $z$-axis are $B_{x j}, B_{y j}$, and $B_{z j}$ which are presented as Eq. (8):

$$
\begin{cases}B_{x j}=0 & (1 \leq j<n) \\ B_{x j}=\frac{\mu_{0}}{4 \pi} \int_{\left(L_{C}\right)} \frac{I_{C} \mathrm{~d} \vec{l} \times \vec{r}}{b^{2}} & (j \geq n) \\ B_{y j}=B_{z j}=0 & \end{cases}
$$

in which $b$ is the distance from detection points to the pathway of current $I_{C}$. $L_{C}$ is the length of current $I_{C}$.

The currents turn to transverse direction below the liquid surface $[5,2,9]$, so $B_{x j}=0(1 \leq j<n)$, and over the liquid surface $B_{x j} \neq 0(j \geq n)$. In this case both $B_{y j}$ and $B_{z j}$ are the constant 0 . Thus, the key parameters, including liquid level and electrode tip, can also be obtained at the point $P_{2}$. 


\subsection{Calculating the spatial sampling interval of detec- tion points}

According to the theory of digital imaging detection technology [26], low density of sampling interval causes the artifact. And the denser sampling interval can achieve the higher spatial resolution and more authentic furnace condition. However, an excess of redundancy leads to a waste of resources. We take the $z$-axis component $B_{z}$ as an example to discuss how to determine the spatial sampling interval. $h_{i}$ and $h_{i+1}$ is the height of the detection point $S_{\mathrm{i}}$ and $S_{\mathrm{i}+1}$ respectively. $B_{z i}$ and $B_{z(i+1)}$ is the $z$-axis component of magnetic flux density at the detection point $S_{\mathrm{i}}$ and $S_{\mathrm{i}+1}$ respectively. In this system, with $\Delta h=h_{(i+1)}-h_{i}$ being the spatial interval, the variation of $B_{z}$ between adjacent points is defined as $\Delta B=B_{z(i+1)}-B_{z i}$. The distribution characteristic of $B_{z}$ and the relation between $\Delta B$ and $\Delta h$ is given in Fig. 8.

In Fig. 8, the tangent line at detection point $S_{\mathrm{i}}$ is $S_{\mathrm{i}} T$. Based on the basic method of local linearization of the nonlinear function in differential calculus [27], in the vicinity of the detection point $S_{\mathrm{i}}$, the tangent segment $S_{\mathrm{i}} P$ can be an approximate substitute for the curved section $S_{\mathrm{i}} S_{\mathrm{i}+1}$, namely $d B$ is approximated as $\Delta B$. Thus, when the derivative of some point $B_{Z}^{\prime}(h) \neq 0$, the relation between $d B$ and $\Delta h$ can be expressed by Eq. (9), where the adjoining points interval $\Delta h<<a$.

$$
|\Delta B| \approx d B=\left|B_{z}^{\prime}(h)\right| \Delta h
$$

$\delta$ is assumed to be the resolution of the magnetic array sensors, and once the hardware development is finished, $\delta$ is a constant. While the variation of $B_{z}|\Delta B|<\delta$, it is beyond the recognition capability of the hardware equipment. Therefore, it is reasonable to take $|\Delta B|=\delta$. So $\Delta h$ can be obtained with Eq. (10) dealing with engineering:

$$
\Delta h \approx \frac{\delta}{\left|B_{z}^{\prime}(h)\right|}
$$

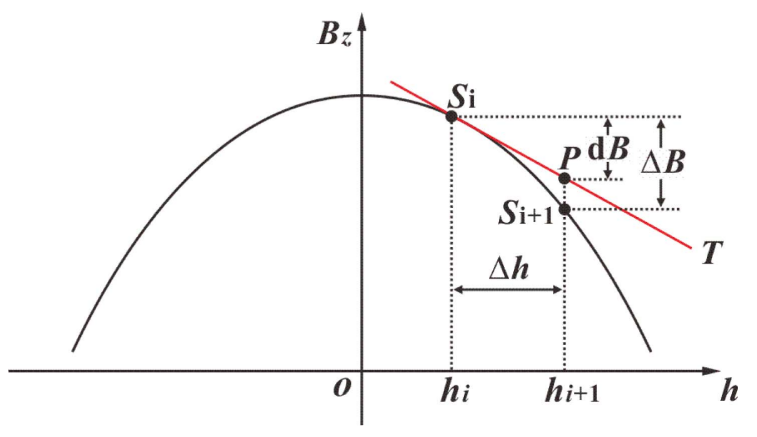

Fig. 8. (Color online) The distribution characteristic of $B_{z}$ and the relation between $\Delta B$ and $\Delta h$.
From Eq. (10), among the detection points $\left(S_{1}, S_{2}, \ldots\right.$, $\left.S_{\mathrm{m}}, \ldots, S_{\mathrm{i}}, \ldots\right)$, the point at which the maximum value of change rate of magnetic induction $\left|B_{z}{ }^{\prime}(h)\right|_{\max }$ locates has strict requirement that spatial sampling interval, $\Delta h$, is equal to the minimal value. In practical application, $d$ is defined as spatial sampling distance which is determined by Eq. (11):

$$
d \leq \Delta h_{\min } \approx \frac{\delta}{\left|B_{z}^{\prime}(h)\right|_{\max }}
$$

With $B_{x j}, B_{y i}$, and $B_{z i}$ of the point $S_{\mathrm{i}}$ given by Eq. (7), it is relatively simple to obtain the change rate of the magnetic flux density using differential calculus with Eq. (12):

$$
\left\{\begin{array}{l}
\left(B_{y}\right)^{\prime}=\frac{\mu_{0} I_{A C} L_{A C}}{2 \pi R r^{2}}\left(\frac{1}{2}-\frac{h^{2}}{r^{2}}-\frac{h^{2}}{2 R^{2}}\right) \\
\left(B_{x}\right)^{\prime}=0 \\
\left(B_{z}\right)^{\prime}=\frac{\mu_{0} I_{A C} L_{A C} a h}{2 \pi R r^{2}}\left(\frac{1}{2 R^{2}}-\frac{1}{r^{2}}\right)
\end{array}\right.
$$

Since the detection points are outside of the furnace, $\mu_{0}$ is equal to $4 \pi \times 10^{-7} \mathrm{H} / \mathrm{m}$, and $I_{A C}$ is approximately equal to $40 \mathrm{kA}$ [5]. According to the environmental observation in real scene, the values of the parameter $a$ and $L$ are set to $a \approx 8.5 \mathrm{~m}$ and $L_{A C} \approx 1.6 \mathrm{~m}$, respectively. We apply the parameters mentioned above to Eq. (12) and calculate the maximal change rate of the magnetic flux density, as Eq. (13) shows below:

$$
\left\{\begin{array}{l}
\left|B_{y}{ }^{\prime}(h)\right|_{\max }=1.04 \mathrm{mGs} / \mathrm{cm} \\
\left|B_{z}{ }^{\prime}(h)\right|_{\max }=0.89 \mathrm{mGs} / \mathrm{cm}
\end{array}\right.
$$

In this paper the resolution of the magnetic array sensors $\delta$ equals $12 \mathrm{mGs}$. we apply the parameters in Eq. (13) to Eq. (10) and Eq. (11), and then the minimum of $\Delta h$ in $y$-axis ans $z$-axis is computed as follows:

$$
\left\{\begin{array}{l}
\Delta h_{y \min } \approx 11.5 \mathrm{~cm} \\
\Delta h_{z \min } \approx 13.5 \mathrm{~cm}
\end{array}\right.
$$

From Eq. (14), when $d \leq 11.5 \mathrm{~cm}$, we can get the required signal. Considering the restrictions in terms of hardware design, the spatial sampling distance $d$ is set to $10 \mathrm{~cm}$.

3.3. The real product structure of magnetic field arrays The magnetic field array with the sensors in series is 


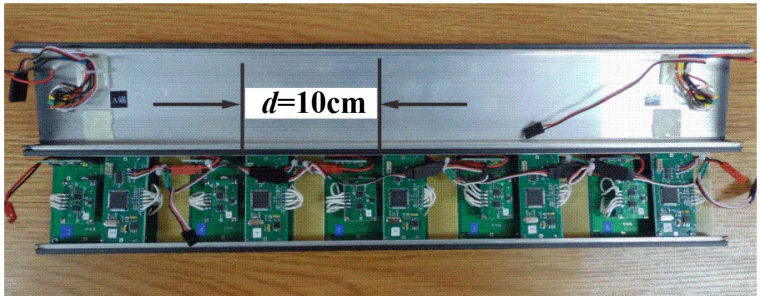

Fig. 9. (Color online) The internal structure of the magnetic array unit.

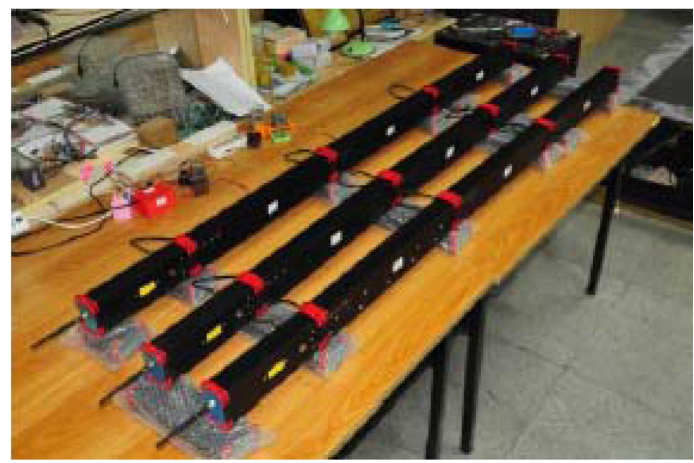

Fig. 10. (Color online) The real product of magnetic field arrays.

composed of some array units, one of which consists of five 3D probes. The internal structure of the magnetic array unit is shown in Fig. 9.

In one array unit, the spatial interval between adjacent $3 \mathrm{D}$ probes is $10 \mathrm{~cm}$. Fig. 10 shows the real product photo of magnetic field arrays.

In Fig. 10, the length of every array is $180 \mathrm{~cm}$ in which the length of power supply and MCU is $30 \mathrm{~cm}$.

\subsection{Experiments in real industrial field}

We perform real measurements on the SAF of ferrosilicon manganese alloy (6300 KVA) at a metallurgical company of Yinchuan city in China. According to the theoretical analysis, the distribution of $B_{y}$ and $B_{z}$ in perpendicular bisector contains the information of liquid level, and the distribution of $B_{x}$ on electrode line also contains the key information of liquid level. Through multiple experiments, we choose the test site between perpendicular bisector and electrode line, and the angle is set to about $20^{\circ}$ between the electrode line and the line connecting the test site and the furnace core. The magnetic array which is about $6 \mathrm{~m}$ away from the lateral wall is vertically placed. The measuring schematic diagram in real industrial field is shown in Fig. 11.

In Fig. 11, we set the origin $o$ beneath the array sensor $S_{1} 10 \mathrm{~cm}$, and the measuring range covers the electrode tip. The experimental scene in industrial field is shown in

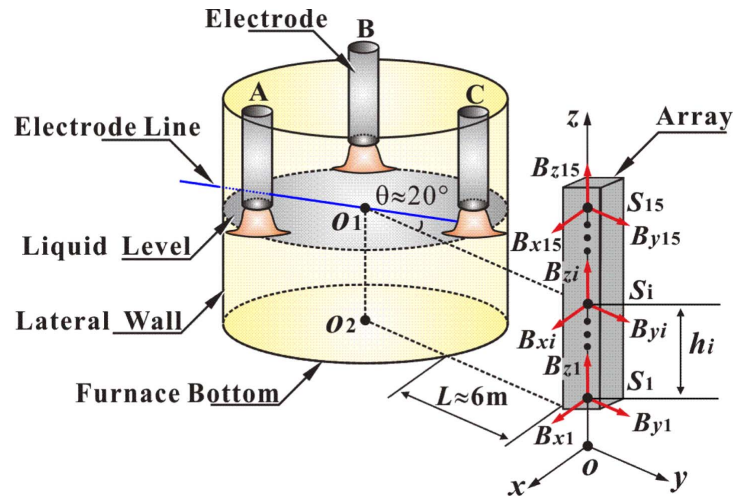

Fig. 11. (Color online) The measuring schematic diagram in real industrial field.

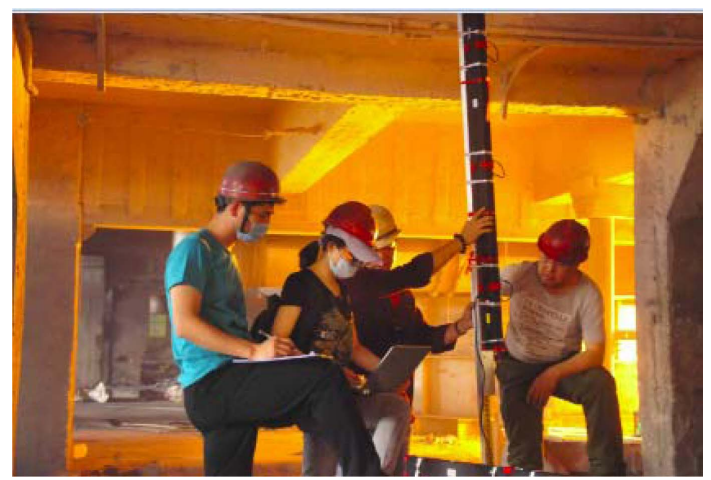

Fig. 12. (Color online) The experimental scene in industrial field.

Fig. 12.

It should be noted that the relationship between the output voltage and the magnetic flux intensity has not been calibrated due to the limit of testing equipment. So we only present measuring results through the output voltage of $V_{x}, V_{y}$, and $V_{z}$.

\section{Results and Discussion}

In the testing experiments, the magnetic flux density components $V_{y}$ of electrode currents and molten currents are obtained at the same time at the detection point. Due to the mixture of the two components, it is difficult to analyze them. Therefore, we only analyze the $h \sim V_{x}$ curve and $h \sim V_{z}$ curve. Moreover, the relative displacement is changed by moving the magnetic sensing array or by lifting and lowering electrodes. The testing results show that the curves of $h \sim V_{x}$ and $h \sim V_{z}$ move towards right while moving the magnetic sensing array downward, and it is shown in Fig. 13.

In Fig. 13, $P_{N}$ is at the peak point of the $h \sim V_{z}$ curve and $G_{N}$ locates the cross point of the curves of $h \sim V_{x}$ and $h \sim V_{z}$ 


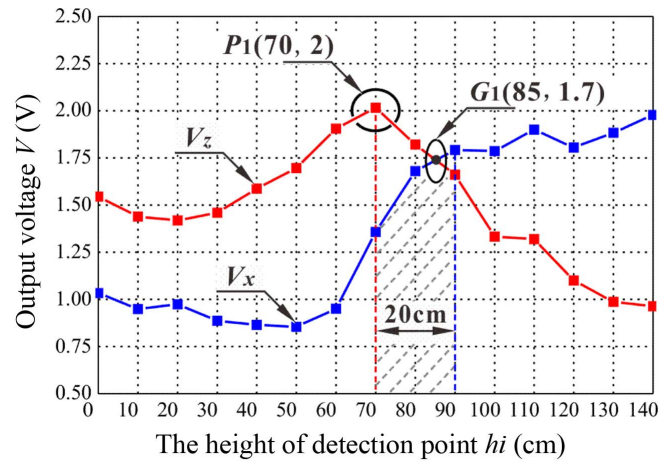

(a) The initial position of the magnetic sensing array

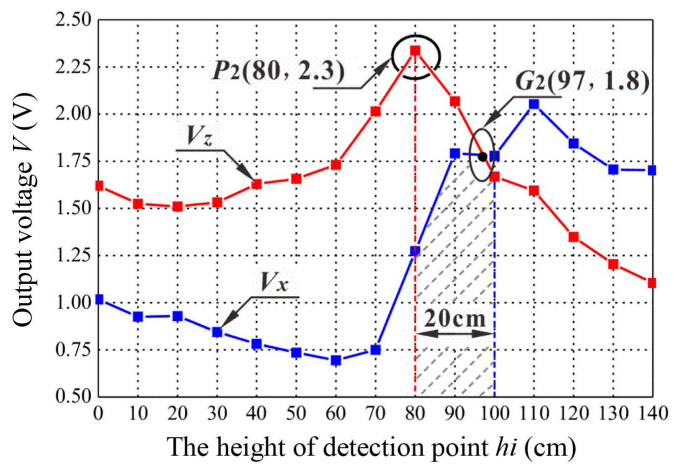

(b) Moving the array downward $10 \mathrm{~cm}$ for the first time

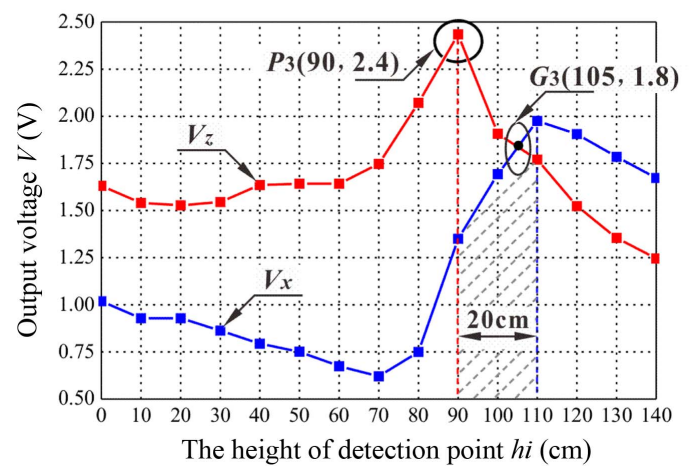

(c) Moving the array downward $10 \mathrm{~cm}$ for the second time

Fig. 13. (Color online) The analysis on testing results.

for $N=1,2,3$. There is a shadow region with the width $20 \mathrm{~cm}$ in Fig. 13. The left side of the shadow region corresponds to the peak point $P_{N}$, and the right side locates near the right of the cross point $G_{N}$. From Fig. 13 it can be found that:

(1) The $h \sim V_{z}$ curve looking like a shape of bell has close correlation to Eq. (7). It can be inferred that the abscissa value of the peak point $P_{N}$ can be regarded as the height of the transverse current $I_{A C}$ that lies beneath the molten bath surface.

(2) The $h \sim V_{x}$ curve shows an monotonous increasing trend in the range of $20 \mathrm{~cm}$ from the beginning with left side of the peak point $P_{N}$ which is about $10 \mathrm{~cm}$ away from $P_{N}$. It reflects the position of the electrode tip and the changes of the current intensity from the liquid level to the electrode tip.

(3) The abscissa value of the cross point $G_{N}$ indicates that the position of $G_{N}$ is near the electrode tip. And at the right of the point $G_{N}$ the $h \sim V_{x}$ curve tends to stable. Based on the above analysis, we can conclude that the right region of the point $G_{N}$ lies above the electrode tip.

(4) In Fig. 13, the difference values of the abscissa at the peak point $P_{N}$ and the cross point $G_{N}$ are both $10 \mathrm{~cm}$. It can be seen that when the relative displacement between the magnetic sensing array and the electrode is 10 $\mathrm{cm}$, the relative displacement of the $h \sim V_{x}$ curve and $h \sim V_{z}$ curve is also $10 \mathrm{~cm}$.

\section{Conclusions}

This paper proposes an array sensing detection method for smelting parameters of SAF based on electromagnetism. Compared with single point 3D detection of magnetic field, the proposed approach has the higher efficiency and the superiority over real-time performance and precision. Theoretical analysis show that if the spatial sampling distance of the magnetic sensing array is set to $10 \mathrm{~cm}$, the distribution characteristics of SAF can be reflected, and some key parameters in the smelting process can be achieved. Moreover, experimental results show that the relative displacement can be measured precisely by the collected information through the magnetic array when the relative displacement between the array and the electrode is $10 \mathrm{~cm}$.

\section{Acknowledgements}

This work was supported by the National Natural Science Foundation of China under Grants 61575137 and Natural Science Foundation of Shanxi Province of China (2014011021-1).

\section{References}

[1] C. X. Li, F. Wang, and J. C. San, Cast Alloy and Melting, Chemical Industry, Beijing (2012) pp. 126-129.

[2] F. Martell, M. Ramirez, A. Llamas, and O. Micheloud, ISIJ International. 53, 743 (2013).

[3] M. Kadkhodabeigi, H. Tveit, and J. S. Tore, ISIJ International. 51, 193 (2011).

[4] F. Martell, A. Deschamps, R. Mendoza, M. Melendez, A. Llamas, and O. Micheloud, ISIJ International. 51, 1846 (2011).

[5] M. Ramirez, J. Alexis, G. Trapaga, and P. Jonsson, ISIJ International 41, 1146 (2001).

[6] V. Logar and I. Skrjanc, ISIJ International 52, 1924 (2012). 
[7] A. C. Mulholland, P. J. Breretonstiles, and C. J. Hockaday, J. S. Afr. I. Min. Metall. 109, 601 (2009).

[8] N. N. Zhang, Z. J. Wang, and D. J. Zhang, IEEE. Comput. Mechatr. Contr. Electr. Eng. 3, 108 (2010).

[9] J. Alexis, M. Ramirez, G. Trapaga, and P. Jonsson, ISIJ. International 40, 1089 (2000).

[10] S. Dwarapudi, V. Tathavadkar, B. C. Rao, T. K. S. Kumar, T. K. Ghosh, and M. Denys, ISIJ International 53, 9 (2013).

[11] T. Ito, J. Yotsuji, and A. Nagamune, ISIJ International 54, 2618 (2014).

[12] Y. L. Wang, Metallurgy of Iron and Steel [M]. Metallurgical Industry, Beijing (2013) pp. 267-269.

[13] J. Zhang, S. J. Chu, and Z. S. Li, Ferro-Alloys 3, 5 (2014).

[14] S. M. Kang, Ferro-Alloys 2, 30 (2012).

[15] J. M. Wu, Q. X. Wang, Z. Q. Xu, and Q. H. Zhu, Contr. Instru. Chem. Indus. 41, 181 (2013).

[16] Y. Wang, Z. Z. Mao, H. X. Tian, Y. Li, and P. Yuan, J. Cent. South. Univ. T. 17, 560 (2010).

[17] Y. Bai, Q. Wang, F. R. Meng, and H. Y. Wang, J. ChangChun. University. T 33, 383 (2012).
[18] Z. H. An, Ms. D. Thesis, Changchun University of Technology, China (2010).

[19] S. J. Chu, S. L. Zeng, and Z. C. Huang, Ferro-Alloys 2, 13 (2009).

[20] S. J. Chu, X. E. Bao, and Z. S. Li, Ferro-Alloys 3, 22 (2013).

[21] A. S. Hauksdottir, T. Soderstrom, Y. P. Thorfinns son, and A. Gestsson, IEEE.T. Contr. Syst. T. 3, 377 (1996).

[22] Q. H. Xiao, Ferro-Alloys 1, 11 (1982).

[23] N. S. Zhang, Ferro-Alloys 6, 1 (1986).

[24] C. C. Liu, Z. C. Jin, and C. J. Xu, J. Agricul. Machi. 25, 28 (1994).

[25] H. Ma and J. B. Wang, Instrument Precision Theory, Beijing University of Aeronautics and Astronautics, Beijing (2014) pp. 364-365.

[26] J. Z. Zhang, Non-destructive Detecting Technology and Application, Science, Beijing (2010) pp. 130-150.

[27] Department of Mathematics of Tongji University. Higher Mathematics, Higher Education, Beijing (2007) pp. 113123. 